InIFolPharm

Alzheimer-Demenz

\section{Bei Antidementiva das Interaktionspotenzial im Auge behalten}

Dass die Gabe mancher Inkontinenztherapeutika sich aufgrund ihrer antichotherapeutika verträgt, ist hinlänglich bekannt. Wenn ein Patient mit Alzheimer-Demenz eine Inkontinenztherapie benötigt, sollte er ein Mittel bekommen, das die Bluthirnschranke nicht passiert, wie etwa Trospium und Fesoterodin, erinnerte Dr. Gabriel Eckermann, Psychiater am Bezirkskrankenhaus Kaufbeuren. Bedeutsam sind aber auch die pharmakodynamischen Wechselwirkungen. Hepatische Interaktionen von Antidementiva mit Substanzen, die über das Cytochrom-P450-System der Leber verstoffwechselt werden, sind vielen Kollegen weniger gut präsent, aber nicht weniger wichtig.

Der Metabolisierungsstatus eines Patienten macht sich vor allem an Polymorphismen von CYP2D6 fest: $10-15 \%$ der Kaukasier sind „slow metabolizer", bei denen 2D6-Substrate rasch akkumulieren. Etwa $7 \%$ zählen zu den „ultrafast metabolizers“, bei denen es oft kaum gelingt, ausreichende Plasmaspiegel aufzubauen. Galantamin und Donepezil werden jeweils zur Hälfte über CYP 2D6 und linergen Wirkung schlecht mit Demenz-
CYP 3A4 verstoffwechselt, sodass jeder Induktor oder Inhibitor dieser Isoenzytiva verändert. Dagegen sind Memantine (z. B. Axura ${ }^{\circledR}$ ) und Rivastigmin keine Substrate von CYP-Enzymen, „Das ist ein wesentlicher Vorteil bei den oft multimorbiden Demenzpatienten“, so Eckermann. Vorsicht ist auch bei Ginkgo biloba geboten, weil es selbst als Induktor am CYP2C19 fungiert. Über dieses Enzym werden manche Antidepressiva, Trizyklika, Benzodiazepine und Protonenpumpenhemmer metabolisiert. Ob ein Patient Ginkgo nimmt, muss der Arzt aktiv erfragen. „Viele geriatrische Patienten erzählen nicht von sich aus, welche ,natürlichen Mittel' sie neben der von uns verordneten Medikation noch nehmen“, warnte Eckermann. In Zweifelsfällen sollten lieber einmal zu oft als zu selten die Plasmaspiegel der psychiatrischen Medikamente gemessen werden.

Manuela Arand, freie Medizinjournalistin

36. Workshop des Zukunftforums Demenz "Medikamentöse Therapie bei der demenziellen Erkrankung - State-of-the-art", Berlin, 28.11.2012. Veranstalter: Merz me die Konzentration dieser Antidemen-

\section{App für Patienten mit Depression}

Das Unternehmen Lundbeck stellt ab sofort die App „Back to Job“ für Patienten mit Depression zur Verfügung. Die Anwendung für das iPhone kann kostenlos im App-Store heruntergeladen werden. Die App unterstützt Patienten bei der regelmäßigen Dokumentation ihrer Gefühlslage, gibt Tipps für den Alltag und bietet die Möglichkeit, die Dokumentation mit dem behandelnden Arzt abzustimmen. Die Patienten können einzelne Parameter der Krankheit nach dem Schulnotensystem bewerten. Beurteilt werden unter anderem die aktuelle Stimmung, das Maß an Freude und Interesse, Energie und Antrieb, das körperliche Befinden und die Schlafqualität. Die Daten werden als Grafik für verschiedene Zeiträume ausgegeben und können per E-Mail an den Arzt gesandt werden. Dieser kann so etwaige Änderungen im Affekt erkennen und die Behandlung des Patienten gegebenenfalls individuell anpassen. Berufstätigen Patienten mit Depression, die ihr Smartphone immer bei sich haben, kann die App zusätzlich helfen, ihren Alltag zu strukturieren. Die App soll in Kürze auch für Android-Systeme verfügbar werden.

Nach Informationen von Lundbeck

\section{Zulassungsantrag angenommen}

Die US-amerikanische Food and Drug Administration (FDA) hat den Zulassungsantrag für Alemtuzumab zur Behandlung der schubförmigen multiplen Sklerose zur Prüfung angenommen. Mit einer Entscheidung wird im zweiten Halbjahr 2013 gerechnet. Genzyme hat seinen Antrag auf Marktzulassung für Alemtuzumab auch schon bei der Europäischen Arzneimittelagentur (EMA) eingereicht, wo das Prüfungsverfahren bereits läuft. Mit einer Stellungnahme des Ausschusses für Humanarzneimittel (CHMP) wird im zweiten Quartal des Jahres 2013 gerechnet.

Nach Informationen von Genzyme, ein Unternehmen von Sanofi 